\title{
Protée
}

\section{Cris et médiations entre les arts : de Lessing à Bacon}

\section{Johanne Lamoureux}

Volume 28, numéro 3, 2000

Mélancolie entre les arts

URI : https://id.erudit.org/iderudit/030600ar

DOI : https://doi.org/10.7202/030600ar

Aller au sommaire du numéro

Éditeur(s)

Département des arts et lettres - Université du Québec à Chicoutimi

\section{ISSN}

0300-3523 (imprimé)

1708-2307 (numérique)

Découvrir la revue

\section{Citer cet article}

Lamoureux, J. (2000). Cris et médiations entre les arts : de Lessing à Bacon. Protée, 28(3), 13-21. https://doi.org/10.7202/030600ar

\section{Résumé de l'article}

À travers sa disqualification sémiotique du cri comme motif de la peinture et de la sculpture, Lessing inaugure la position moderniste qui postulera la séparation des médiums en autant de domaines autonomes. En amont et en aval de cet interdit, le présent article analyse quelques oeuvres peintes qui paraissent confirmer que le cri entraîne un brouillage des médiums. Et certains artistes semblent avoir représenté le cri afin, précisément, de jouer sur ce glissement. À partir des papes hurlants de Francis Bacon, où s'enchevêtrent des références et des matériaux empruntés à la peinture, à la photographie et au cinéma, nous distinguerons deux usages de la médiation photographique : d'une part, en tant que la photographie introduit dans les processus de fabrication de l'image un contact distancié entre le peintre et le modèle, entre le sujet et l'objet et, d'autre part, en tant qu'elle est, selon la formule de l'artiste, transformée en " humus " par l'usure qu'elle connaît dans l'atelier du peintre, la médiation photographique sert paradoxalement à confirmer l'homogénéité picturale de l'image produite.
Ce document est protégé par la loi sur le droit d'auteur. L'utilisation des services d'Érudit (y compris la reproduction) est assujettie à sa politique d'utilisation que vous pouvez consulter en ligne.

https://apropos.erudit.org/fr/usagers/politique-dutilisation/ 


\section{CRIS ET MÉD IATIO N S EN TRE LES ARTS: DE LESSING À BACON}

JOHANNE LAMOUREUX

La réflexion sur l'intermédialité a depuis 1997 commencé de se regrouper autour d'un centre - Centre de recherche sur l'intermédialité - où se poursuivent divers travaux qui étudient, selon la définition d'André Gaudreault, «le procès de transfèrement et de migration, entre les médias, de formes et de contenus » ${ }^{1}$. Il m'a paru curieux d'observer que le CRI, acronyme sous lequel ce centre s'affiche, renvoie par homonymie à un référent dont l'histoire de l'art et l'esthétique de la fin du dix-huitième ont dénoncé la traduction plastique au nom précisément d'une incompatibilité fondamentale entre certains thèmes et certains médiums. Le cri aura donc été un temps l'emblème d'une confusion entre des arts fantasmés comme des mondes autonomes et étanches, comme des «monades $»^{2}$ : le voici désignant désormais un ensemble de recherches qui visent à réfléchir les articulations historiques et théoriques au sein de la production culturelle, dans le contexte actuel d'un incontournable arrimage technologique de tous les médias.

S'arrêtant dans un premier temps à la totale disqualification sémiotique du cri plastique par Lessing ${ }^{3}$, le présent essai commentera ensuite, en amont et en aval de ce moment historique, certains des croisements interdisciplinaires auxquels ce motif a pu donner lieu, en insistant tout particulièrement sur le travail du peintre Francis Bacon. L'exemple de Bacon est intéressant à au moins deux égards: d'une part, parce qu'il prend place à l'époque où, de l'autre côté de l'Atlantique, la critique formaliste américaine commence à se formuler à partir d'un retour aux principes de Lessing; et, d'autre part, parce que l'intérêt du peintre pour le cri en peinture est bien documenté et manifeste un important travail sinon d'intermédialité, du moins de médiation entre les arts, travail que la prédominance des lectures phénoménologiques de son œuvre et la conception immédiate de l'image qu'elles favorisent ont eu trop tendance à occulter jusqu'ici.

En évoquant l'exemplarité du cri dans l'esthétique du XVIIIe siècle, je fais allusion bien sûr au traité Laocoon, rédigé en 1766 par Gottfried Ephraim Lessing ${ }^{4}$. Dans cette attaque contre la doctrine de l'ut pictura poesis, Lessing réfléchit au domaine propre à chaque art et tente de distinguer les contraintes ontologiques des arts du temps, comme la poésie, de celles des arts de l'espace, comme la sculpture: il mène sa démonstration à partir des écarts dans la 
représentation de la souffrance du prêtre Laocoon, selon que celle-ci apparaisse dans L'Énéide de Virgile ou dans le légendaire groupe sculptural du IIe siècle avant Jésus-Christ où, à la différence du long cri évoqué par le poème, l'artiste a traduit la douleur par un gémissement qui entrouvre à peine les lèvres du protagoniste et leur conserve un contour assez régulier. Le traité de Lessing, pour incontournable qu'il soit aux yeux des spécialistes du XVIIIe siècle, pourrait bien n'incarner qu'une spéculation esthétique sans grande conséquence dans l'histoire, mais il n'en est rien dans la mesure où il servit à étayer les fondements théoriques et historiques du formalisme de l'après-guerre, tels qu'on les trouve formulés dans les écrits du critique américain Clement Greenberg, et tout particulièrement dans son article de 1940 titré "Towards a Newer Laocoon " 5 . Greenberg y résume le projet de l'avantgarde artistique en l'inscrivant dans la mouvance de Lessing, mais une mouvance revue et corrigée, puisque l'auteur allemand postulait, lui, une suprématie des arts du temps ${ }^{6}$. Gardant toutefois l'idée d'un domaine propre à chaque art, le critique américain formulera, en écho à ce texte, sa théorie de la spécificité du médium et ses nouvelles exigences, établissant que la réussite de l'avant-garde réside dans sa prise en compte de, voire dans sa reddition à la résistance du médium, c'est-à-dire pour la peinture, comme il le précisera plus tard, dans la planéité et les délimitations de la planéité. Cette filiation moderniste de Lessing à Greenberg a elle-même été analysée, dans un essai de Thierry de Duve intitulé «Clement Lessing» 7, au moment où, en 1979, le formalisme du critique américain était définitivement mis en crise et dépassé par la nouvelle indécidabilité catégorielle des pratiques artistiques contemporaines.

En somme, pour une perspective interdisciplinaire, l'importance des théories de Lessing s'est trouvée réactivée, au fil du XXe siècle, du fait qu'elles aient été construites après-coup comme le moment fondateur d'une conception du modernisme, selon laquelle la seule réflexivité critique valable est celle qui fait l'économie de tout élément étranger à la définition essentialiste d'un médium et qui restreint son développement à la stricte exploration des conditions de possibilité limites de ce médium. Il semblerait que l'insertion du cri au sein de la représentation plastique vienne troubler cette étanchéité des pratiques: ce postulat apparaît tant à travers l'exemple retenu par Lessing qu'à travers les contre-exemples picturaux qu'il omet de mentionner et à travers le procès interdisciplinaire qui engage une résurgence du motif du cri chez Bacon, parallèlement à la formulation du modernisme de Greenberg.

C'est donc dire que je ne remettrai pas en cause ici la validité de la séparation, défendue par Lessing, entre les arts du temps et les arts de l'espace. W.J.T. Mitchell a déjà bien analysé la relative inopérativité de ce cloisonnement et sa constante transgression par la pratique. La distinction entre les arts du temps et de l'espace représentait une opposition relevant déjà du lieu commun à l'époque de Lessing, mais personne, avant ce dernier, n'avait proposé de l'ériger en principe esthétique normatif, régulant ce qui est acceptable ou non selon les contraintes du genre pratiqué. Comme l'écrit Mitchell, la thèse de Lessing participe du déni, car «il n'y aurait point besoin de dire que les genres ne doivent pas être mélangés, s'ils ne pouvaient pas être mélangés » ${ }^{8}$.

Cri: «son perçant émis par la voix [...] paroles prononcées très fort sur un ton aigu [...] mouvement intérieur de la conscience [...] son émis par les animaux, variant avec les espèces» et enfin «bruit aigre et peu harmonieux".

Les définitions du cri du Robert éclairent bien le problème posé dans le cadre d'une séparation des arts selon les catégories de l'espace et du temps: avant de révéler un problème esthétique, la traduction plastique du cri ouvre d'entrée de jeu sur une dimension interesthésique: donner à voir, dans l'espace, ce qui se donne à entendre, dans la durée. De plus, le cri se situe au nœud d'une difficulté tout à fait bien entrevue par Lessing lui-même: si les arts de l'espace doivent se consacrer à la représentation des corps et les arts du temps à la représentation des 
actions, il n'en demeure pas moins que les corps existent eux aussi dans le temps. La peinture pourra donc imiter des actions, soutient Lessing, à condition que celles-ci soient suggérées par la forme. Cette réserve ne surprend pas, mais elle indique précisément en quoi, particulièrement dans le contexte du néo-classicisme européen, la représentation du cri constitue un scandale plastique: elle introduit dans l'image une plage de dé-formation.

Le plaidoyer de Lessing contre la représentation du cri en peinture et en sculpture va, au fil des pages du traité, asseoir son rejet sur chacune des dimensions sémiotiques du motif qu'il dénonce, mais c'est d'abord sur le plan syntaxique, en tant que le cri vient rupturer l'image, y introduire une zone allotopique qui se soustrait aux exigences étroitement canoniques du beau néo-classique, que le groupe sculptural du Laocoon est loué d'avoir évité le cri franc qui défait immanquablement l'harmonie du visage (cette rupture redoublant le manque d'harmonie qui définit, on l'a vu, le cri dans le domaine sonore):

L'artiste voulait représenter la beauté la plus grande compatible avec la douleur physique. Celle-ci, dans toute sa violence déformatrice, ne pouvait s'allier avec celle-là. L'artiste était donc obligé de l'amoindrir, de modérer le cri en gémissement, non pas parce que le cri indique une âme basse, mais parce qu'il donne au visage un aspect repoussant. Imaginez Laocoon la bouche béante et jugez. [...] Une bouche béante est, en peinture, une tache, en sculpture un creux, qui produisent l'effet le plus choquant du monde, sans parler de l'aspect repoussant qu'elle donne au reste $d u$ visage tordu et grimaçant. [...] Quand le Laocoon de Virgile crie, à qui vient-il à l'esprit que, pour crier, il faut lui élargir la bouche et que cette bouche déformée le rend laid? Il suffit que le clamores horrendos ad sidera tollit soit un trait magnifique pour l'oreille, il peut être ce que l'on voudra par rapport au visage. [... $]^{9}$

Puis, se déplaçant sur un autre terrain, l'argumentation de Lessing motive aussitôt l'exclusion du cri par des considérations qui ressortissent cette fois du niveau sémantique; elle met en garde contre le portrait psychologique et éthique du modèle qui, dans les arts plastiques, ouvrirait la bouche jusqu'au cri:
Le Laocoon de Virgile crie, mais ce Laocoon qui crie, est le même que nous connaissons et que nous aimons déjà comme le patriote le plus clairvoyant, comme le père le plus affectueux. Nous attribuons son cri, non à son caractère, mais uniquement à son intolérable souffrance. C'est elle seule que nous entendons dans son cri, et c'est par ce cri seul que le poète pouvait nous la faire comprendre. Qui pourrait l'en blâmer? Ne doit-on pas plutôt reconnaître que, si l'artiste a bien fait en ne faisant pas crier Laocoon, le poète aussi a bien fait en le faisant crier? ${ }^{10}$

On notera qu'entre des remarques étayées sur la syntaxe de l'œuvre (préserver la loi de la beauté dans le rapport de la bouche au visage) et celles qui s'appuient sémantiquement sur la nécessité de rendre le caractère exemplaire du modèle, la question du temps a fait son apparition. Les observations sur la tache ou le creux manifestaient un refus de ce qui peut troubler la cohérence spatiale de l'œuvre, celles sur Laocoon, patriote clairvoyant et père affectueux, donnent cette fois à entendre que l'œuvre plastique est limitée du fait qu'elle ne dispose que d'un «instant unique», qui empêche le regardeur d'avoir accès à d'autres facettes du personnage venant nuancer, excuser la démonstration de son extrême douleur au moment où les serpents les menacent, lui et ses fils.

La reconnaissance de cet instant unique entraine finalement Lessing vers une prise en compte de la pragmatique du cri pictural, où comptent moins la cohérence de l'œuvre et l'exemplarité du héros que leurs effets sur le spectateur:

Si l'artiste ne peut jamais saisir qu'un seul instant de la nature toujours changeante; si, en outre, le peintre ne peut utiliser qu'un unique point de vue pour saisir cet unique instant; si, d'autre part, ses œuvres sont faites pour être non seulement vues, mais contemplées longuement et souvent, il est alors certain que cet instant et ce point de vue uniques ne sauraient être choisis trop féconds. Or cela seul est fécond qui laisse un champ libre à l'imagination. Plus nous voyons de choses dans une ouvre d'art, plus elle doit faire naître d'idées; plus elle fait naître d'idées, plus nous devons nous figurer y voir de choses. Or dans le cours d'une passion, l'instant du paroxysme est celui qui jouit le moins de ce privilège. Au-delà, il n'y a plus rien, et présenter aux yeux le degré extrême, c'est lier les ailes à l'imagination. Ne pouvant s'élever 
au-dessus de l'impression sensible, elle doit se rabattre sur des images plus faibles et craindre de se limiter à ce qui lui apparaît dans la plénitude du visible. Si Laocoon gémit, l'imagination peut l'entendre crier; mais s'il crie, elle ne peut ni s'élever d'un degré, ni descendre d'un degré de cette image sans le voir dans un état plus supportable, donc moins intéressant. Ou elle l'entend seulement gémir, ou elle le voit déjà mort. 11

Rupture et opacification matérielle de l'œuvre, discrédit du modèle, frein à l'imagination du spectateur, autant de raisons différentes d'exclure le cri de la représentation plastique et d'en réserver l'emploi au poète (au poète épique et pas même au poète dramatique puisque la représentation scénique doit le proscrire aussi). Lessing se donne donc beaucoup de mal pour soutenir que les Anciens l'ont scrupuleusement évité, même dans leur période décadente, et qu'il est impossible que l'Ajax peint par Timomaque ait hurlé. Sur les Modernes et le cri, Lessing est silencieux. Rare, il est vrai, dans la peinture de la Renaissance et des siècles suivants, le cri n'y a pourtant pas été victime du même interdit absolu avant Lessing.

Que faire par exemple, à la lumière de l'interdiction néo-classique de Lessing, de la Méduse de Caravage, une autre histoire de serpents? On pourrait dire bien sûr que, dans le cas de la Méduse, la fonction apotropaïque l'emporte sur la fonction narrative, que la captation de l'image par le bouclier qui doit effrayer l'ennemi excuse la représentation d'un tel moment de paroxysme, bien que, ainsi que Louis Marin l'a finement analysé, l'instant représenté par cette œuvre participe d'une temporalité complexe. La tête déjà tranchée par Persée, le héros absent, semble avoir été coupée au moment même où son regard révulsé, piégé par le reflet, cherchait à se fuir dans la direction opposée de son cri, qu'il faut supposer muet, interrompu par la lame. Marin écrit:

Le Caravage saisit cet instant de la pétrification, si l'on peut dire, un peu avant que la pétrification ne se produise. Le moment le plus fugitif, l'infinitésimal de temps, de durée, est cependant, dans son évanescence même, l'instant le plus durable, le plus permanent. L'instant sculptural. 12
Voilà donc mis au jour une hybridation plastique, jouée au sein même des arts de l'espace, dans le défi qui consiste à rendre en peinture le moment d'un devenir-statue de la Gorgone. La question de la temporalité ne pose pas problème puisqu'il s'agit moins de condenser un temps moyen (celui qui sépare la pétrification de la décapitation) que de traduire la complexité de l'instant d'une conversion fatale de la chair en pierre. Mais il ne faudrait pas limiter cette rencontre entre deux médiums à une simple référence de la peinture à la sculpture. Après tout, la représentation de Méduse s'auto-pétrifiant implique non seulement la réitération d'un motif, mais celle du support objectal où ce motif se retrouve traditionnellement: le tableau imite l'image de la Gorgone et l'objet-bouclier.

Il est intéressant d'observer que Louis Marin, préoccupé d'analyser le tableau du Caravage dans sa dimension de "piège-à-regard", ne commente guère le cri qu'on y trouve, et ce même dans l'intermède psychanalytique où il aborde le thème de l'œuvre dans la perspective freudienne du fétichisme. Insistant sur la chevelure serpentine de Méduse et sur le coup d'œil maléfique auxquels répond la décapitation castratrice, Marin passe sous silence le fait que la structure fétichiste procède non seulement d'un déni de la différence sexuelle de la femme, à travers le déplacement et la fixation sur des attributs phalliques, mais que ce déni implique aussi, et d'abord, une reconnaissance de cette différence, différence que le cri, tache et trou dans le tableau-bouclier, vient presque trop exemplairement figurer ici par dé-figuration.

Toutefois, cette bouche hurlante, stoppée net dans sa tentative de détournement, présente une déformation ironique dans la mesure où celle-ci, loin de rompre cette fois l'harmonie du visage, s'insère et s'accorde pour ainsi dire avec la torsion qui le travaille de part en part et elle s'intègre à la révulsion de la face entière de Méduse, qui cherche ainsi à ne pas se voir dans le reflet où elle s'est déjà reconnue. Il n'y a pas là de quoi réconcilier Lessing avec ce tableau. Après tout, Lessing aurait partagé l'opinion de Bellori selon laquelle Caravage était né, ainsi que le rappelle le titre 
du livre de Marin, pour «détruire la peinture». De plus, le cri peint par Caravage est celui de la Méduse et il a par cela même partie liée avec ce que le critique allemand cherchait à conjurer dans les arts de l'espace: le féminin. «Dans l'imitation matérielle de l'art, écrit-il, l'apparente persistance du cri suffirait à elle seule pour faire de ce cri un signe de faiblesse efféminée» ${ }^{13}$.

On retrouve ici la sourde opposition que Mitchell a exposée chez Lessing entre la création virile des Anciens et les accouchements monstrueux des mères modernes:

Si une belle génération d'hommes produit de belles statues, cellesci à leur tour agissent sur ceux-là, et l'État a dî en partie la beauté de ses hommes à ces ouvres. Chez nous, il semble que la tendre imagination des mères n'est impressionnable que lorsqu'il s'agit d'accoucher de monstres. ${ }^{14}$

Élargissant la portée esthétique du traité de Lessing aux considérations sociosexuelles (gender) et politiques (territoire national) qui le sous-tendent, la lecture de Mitchell fait ressortir une série d'oppositions systématiques, qui règlent la logique de ce texte pourtant décousu dans sa genèse et son déroulement. Ainsi la peinture s'y oppose à la poésie, comme l'espace au temps, les signes naturels aux signes arbitraires, l'imitation à l'expression, le corps à l'esprit, l'extériorité à l'intériorité, le silence à l'éloquence, la beauté au sublime, l'œil à l'oreille et le féminin au masculin. Cette déclinaison d'oppositions, familière chez plusieurs contemporains de Lessing et dont l'expression la plus succincte surgira, comme le suggère encore Mitchell, dans le vers de Blake «Time is man. Space is woman", cette déclinaison donc, qui se clôt sur le gender se prolonge aussitôt dans un rapport aux genres ${ }^{15}$. Apparait alors dans le texte de Lessing une opposition entre le mélange moderne des genres et les genres distincts de l'Antiquité, les monstres modernes et les corps parfaits de l'Antique, les mères et les pères, le raffinement français et la virilité allemande et anglaise.

Dans cette perspective, inscrite entre peinture et sculpture, entre le motif que Lessing interdira à la poésie (le bouclier) ${ }^{16}$ et celui qu'il interdit aux arts de l'espace (le cri), la bouche ouverte de la Méduse apparait comme un véritable emblème de ce qui est à rejeter chez les Modernes selon Lessing: elle combine le féminin, le monstrueux, l'hybride, le nonspécifique. Son cri, cet étranglement sonore qui n'appartient ni au silence des arts de l'espace ni à l'éloquence des arts du temps vient renforcer le principe féminin déjà projeté par Lessing dans les arts de l'espace, ce féminin que Laocoon, dans ce que Mitchell appelle une peur de l'imagerie ${ }^{17}$, vise à refouler en resserrant les lèvres de tous ces héros antiques chargés d'insuffler, comme Philoctète, un peu de virilité en peinture. Il ne faut pas se surprendre que ce qu'on a qualifié de «meilleur cri humain en peinture ${ }^{18}$ soit aussi celui d'une autre femme, une mère hurlante du Massacre des Innocents de Poussin ni de ce que l'auteur de ce jugement soit Francis Bacon.

Nourrie dès le milieu des années trente par diverses sources visuelles, la recherche picturale de Francis Bacon autour du cri s'est longuement élaborée durant la décennie suivante, celle-là même que Clement Greenberg allait inaugurer en voulant relever le projet de Lessing. Le critique américain s'apprêtait ainsi à cerner le paramètre du médium opaque et résistant comme critère essentiel du modernisme et à exclure, cette fois au nom du modernisme et non plus des Anciens, le féminin, le monstrueux, l'hybridation des médiums. Affirmant la spécificité du médium, la distinction de Greenberg ferait une meilleure part aux arts de l'espace, notamment à la peinture (d'où le renforcement d'une rhétorique critique accumulant autour de la toile les métaphores virilisantes) ${ }^{19}$. Durant ce temps, dès l'après-guerre, Bacon, délaissant le problème de l'expression cher à Lessing, s'intéresse au cri par cela même que l'auteur allemand rejetait : la déformation matérielle (la tache, le creux) de l'œuvre, sans égard pour tout relent existentialiste ou toute motivation expressionniste. Rien ne sera plus éloigné des cris peints par Bacon que Le Cri de Munch qui paraît, lui, chercher à traduire, selon les préoccupations interesthésiques ou synesthésiques du symbolisme, les ondes et les réverbérations du son par la ligne qui 
unifie le motif iconique et son traitement graphique. On a beaucoup écrit sur la particularité du cri chez Bacon: principe d'une déformation de la tête en tension avec l'enfermement parfaitement circulaire des protagonistes en piste ${ }^{20}$; soumission exploratoire aux forces qui éloignent du spectacle et rapprochent de la sensation ou stratégie liée à l'élaboration matérielle, entre forme et figuration, d'une peinture de la figure, du figural ${ }^{21}$, motif sexuel portant toute la charge du sado-masochisme de l'artiste ${ }^{22}$. Mais nul n'a mieux parlé que le peintre lui-même de la fascination que le cri exerça sur lui pendant près d'une vingtaine d'années. Bacon soutient avoir voulu «peindre le cri plutôt que l'horreur", avoir tenté à son tour de réaliser «le meilleur cri humain de la peinture»: En conversation avec David Sylvester, il déclare:

J'ai toujours été très ému par les mouvements de la bouche et par la forme de la bouche et des dents. Les gens disent qu'elles ont toutes sortes d'implications sexuelles et j'étais toujours très obsédé par l'apparence même de la bouche et des dents. J'aime, pourraiton dire, le luisant et la couleur qui viennent de la bouche et j'ai toujours espéré, en un sens, être capable de peindre la bouche comme Monet peignait un coucher de soleil. 23

On ne voit donc pas la pertinence qu'il y aurait à tenter d'interpréter ici le motif du cri chez Bacon. Je voudrais donc me contenter de réfléchir ici à la façon dont le cri le plus célèbre de son œuvre semble mettre en jeu un procès entre les arts, qui esquive l'hybridité que les regardeurs actuels attendent désormais d'une telle interrelation.

Inspiré par Poussin et Monet, le cri dans l'œuvre de Bacon apparaît sous l'égide de la peinture, greffé comme il l'est au cœur d'une citation du Portrait $d u$ pape Innocent $X$ peint par Vélasquez trois cents ans auparavant. Michael Peppiatt a rapproché le cri ajouté par Bacon au pape de Vélasquez à l'acte par lequel Duchamp, un artiste fort admiré de Bacon, avait dessiné une moustache à la Joconde 24 . Ce rapprochement résonne singulièrement en ce que non seulement les greffes de ces deux artistes ciblent la même zone orale, mais en tant qu'elles tablent toutes deux sur un effet de travestissement (cross-gendering serait plus juste) que la pourpre et les dentelles du pape viennent encore exacerber dans le cas du Bacon. Or ce cri, on le sait et l'artiste ne s'en cachait pas, Bacon ne l'a pas inventé: il l'emprunte aussi, comme la toile de Vélasquez, mais en recourant cette fois à une source non picturale, en s'échappant du côté du cinéma où il vole à Eisenstein le cliché de la nurse dans Le Cuirassé Potemkine, un film que Bacon vit pour la première fois en 1935 , à peu près à la même époque où il se procura à Paris un livre médical intitulé Diseases of the Mouth dont les illustrations, rehaussées de couleurs, avaient retenu son attention ${ }^{25}$. Ainsi le still cinématographique ne sera pas la seule source du cri papal: aux illustrations scientifiques et à la citation cinématographique, il faut encore ajouter la photo de presse dont la nouvelle rhétorique «sensationnaliste» (sans clin d'œil ici vers la «logique de la sensation" formulée par Deleuze autour du peintre britannique) captivait Bacon: dans son atelier, une de ses nombreuses reproductions photographiques du Vélasquez (Bacon les collectionnait) voisinait avec une photo de journal représentant un Goebbels défiguré par une prestation oratoire.

Le peintre du cri se révèle donc un farouche consommateur d'images, catégorie sous laquelle il cherche d'ailleurs à inscrire sa pratique, se proclamant simplement «image-maker». Outre des emprunts photographiques éclectiques, Bacon réalisera aussi ses portraits à partir de photos, prises pour lui par John Deakins, et il les consultera souvent pendant les séances de pose, souvent même en lieu et place de ces séances, quand il ne va pas jusqu'à consulter au milieu d'une session de travail la photo de quelqu'un d'autre que son modèle.

Je voudrais souligner ici le double statut du rôle médiateur de la photographie dans le travail de Bacon. D'une part, comme l'indique John Russell, le peintre utilise la photo d'une manière qu'il faut inscrire aux antipodes de l'exemple duchampien cité plus haut. La photo n'est jamais pour lui un matériau, intégré comme tel au travail pictural. Il n'y a pas chez Bacon d'emploi ready-made du cliché photographique. Bacon travaille à partir de la photographie. Mais on ne 
saurait trop insister sur l'importance de cette médiation et sur les modalités complexes selon lesquelles le peintre la pose. Sa boulimie d'images scientifiques, journalistiques ou de reproductions artistiques n'a certainement pas à voir simplement avec une disponibilité des sources. À cet égard, son refus, lors de séjours romains, d'aller voir le Vélasquez à la galerie Borghèse ${ }^{26}$ doit être situé dans un contexte élargi. Cette attitude, qu'on pourrait imputer à la croyance de Bacon en une aura de l'œuvre à laquelle il souhaiterait se soustraire, doit plutôt être rapprochée de son insistance à avoir des photos des modèles qu'il utilise pour peindre, allant même à utiliser celles-ci pendant que le modèle s'efforce de maintenir la pose, hors du regard du peintre, attaché, lui, à fixer une des photos de Deakins ou une photo d'un tout autre modèle (par exemple, un hippopotame) dont un aspect (souvent une texture) semble le retenir davantage. La photographie s'apparente ici à une médiation, entendue comme filtre, relais, écran: elle est une mesure prise contre la possibilité de tout contact im-médiat entre le peintre et ses référents, objet ou personne. Contrairement à la situation qui intéresse Silvestra Mariniello, à partir des réflexions de Wlad Godzich sur la reproduction mécanisée du réel, le recours de Bacon à la photographie «n'amène pas le référent dans l'imaginaire en abolissant toute distance » 27 dès lors qu'on ré-inscrit cette modalité de la production du peintre par rapport à ce qui me semble en être le pendant symétrique lors de la réception: en effet, la décision du peintre d'avoir recours, dans ses expositions, à de lourds encadrements dorés et à un verre épais, semble une tentative de souligner, à l'autre bout de la chaîne, un écart similaire entre l'œuvre et le spectateur. Dans cette perspective, la photographie apparaît à chaque fois comme un médiateur des rapports entre sujet et objet, une "distance qui fait lien ${ }^{28}$ ou plus précisément un lien fondé sur la distance plutôt que sur le postulat d'une «chair du monde» où s'abolirait la dualité sujet-objet (et on comprend que le parti pris phénoménologique qui travaille, jusque chez Deleuze, la fortune critique de Bacon n'ait guère favorisé la mise en lumière de cette facette du rôle de la photographie). Si la photo n'est pas ici abolition de la distance, il ne convient pas d'en faire non plus une instauration de distance, mais il y aurait lieu de constater que la photo sert à négocier ponctuellement la distance inhérente à la séance de pose (ou à la contemplation du tableau).

Néanmoins, à côté de cette fonction médiatrice de la photographie, la fabrication des tableaux de Bacon convoque ce médium selon des visées plus fusionnelles, qui touchent à une espèce de phagocytage que le peintre résume magnifiquement dans la citation suivante:

Je me considère comme une espèce de machine pulvérisatrice dans laquelle est introduit tout ce que je regarde et tout ce que je sens. Je me crois différent de ces vautours mass média qui utilisent les photographies plus ou moins telles qu'elles sont, ou qui les découpent pour les arranger différemment. La réalité littérale de ces photographies ainsi employées - même si ce ne sont que des fragments - empêchera l'apparition de véritables images, parce que l'essence des apparences n'a pas été suffisamment digérée et transformée. Dans mon cas, les photographies deviennent une sorte d'humus à partir duquel les images émergent de temps à autre. Les images peuvent être en partie conditionnées par la teneur des matériaux introduits dans le pulvérisateur. 29

Avant de réfléchir au sens figuré de cette déclaration, il importe de la prendre un peu au pied de la lettre. Une série de photographies, réalisées par Sam Hunter de l'atelier de Bacon et de quelques-uns des documents qu'il utilisait, permet de constater la quasi-décomposition des photographies, piétinées, souillées, déchirées, qui occupaient le sol des espaces de travail du peintre. L'humus et la pulvérisation dont le peintre parle ne peuvent pas être ramenés à une figure de style. Ses sources photographiques, dispersées dans un chaos impressionnant, ont été profondément transformées par les conditions de l'atelier, qui agit de la sorte comme un médiateur supplémentaire ${ }^{30}$. Certaines photographies retrouvées laissent à peine voir encore leur référent, sérieusement opacifié par les craquelures, les taches et parfois même 
l'effritement du support. Il y aurait donc là une manière de réfuter la force du préjugé référentiel envers la photographie, laquelle semble dès lors n'avoir agi que comme un aide-mémoire, un nœud porté au doigt. Mais surtout, cet humus évoque la réduction de la photographie à une pulpe que la pâte picturale assimile afin qu'il n'en reste plus de trace dans le tableau final. (On ne sera pas surpris d'apprendre qu'une des photos utilisées par Bacon et que les images de Hunter ont rendu accessibles est le document, extrêmement «travaillé» par les conditions de l'atelier, qui montre Goebbels vociférant.) S'il y a abolition d'une distance ici, il s'agit moins de celle entre un sujet et un objet que celle qui tenterait de réguler en registres distincts la photographie et la peinture comme médiums. La photographie est alors à entendre comme une médiation dont la matérialité est bientôt digérée par une peinture certes réinscrite dans la catégorie des images, mais d'images apparemment toujours homogènes.

Ainsi entendue, cette seconde forme, plus fusionnelle que distanciatrice, de la médiation photographique dont procède le cri pictural chez Bacon nous révèle qu'autour de l'hybridation et du mélange des médiums, la modernité du peintre se distingue du modernisme de Greenberg, héritier de Lessing, dans la seule mesure où, tous deux réfractaires à la narrativité de l'image, le critique américain interdit et refoule l'interdisciplinarité alors que le peintre anglais la dénie, la reconnaissant certes dans les récits autorisés sur sa production mais sans que l'homogénéité matérielle de ses œuvres en soit ébranlée. Dans le tableau final, la poiêsis paraîtra relever de la peinture pure et le procès interdisciplinaire qui en constitue cependant la principale modalité sera recouvert. Pourtant, ici et là, l'humus photographique continue de travailler, de saper l'homogénéité du pictural, car les critiques de Bacon ont sans cesse été préoccupés de rechercher des traces et de nommer les effets, dans le tableau, d'une médiation photographique dont il faudrait par conséquent reconnaître la participation résistante. Durant les années cinquante, les commentaires du Times reviennent inlassablement sur la dette de Bacon envers la photographie et le cinéma, et ils insistent toujours pour en cerner des effets parasitaires dans le tableau: texture désagréable de l'agrandissement photographique, extension temporelle du cinéma (dans les triptyques dont Bacon use, en partie, pour contrer toute narration), effet de projection sur la toile d'un contenu sensationnaliste rappelant la photo de presse et transformant les tableaux, toujours selon les critiques, en "un cri de souffrance» 31 ). Ce serait donc moins le cri qui invite à un brouillage entre les arts que ce brouillage même que l'image de Bacon parvient à produire sous la figure du cri. 


\section{N O TES}

1. A. Gaudreault, «Le cinéma entre intermédialité et littérarité », Du littéraire au filmique. Système du récit, Québec, Paris, Nota bene/ Armand Colin, coll. «U», 1998, p. 169-183.

2. Sur l'artificialité de l'opposition entre arts du temps et arts de l'espace, voir W.J.T. Mitchell, «Space and Time. Lessing's Laocoon and the Politics of Genre ", Iconology, Chicago, The University of Chicago

Press, 1986, p. 95-115. Voir aussi J. Müller, «Top Hat», Cinémas, vol. 5 nos 1-2, automne 1994, p. 213: «Si nous entendons par "intermédialité" qu'il y a des relations médiatiques variables entre les médias et que leur fonction naît entre autres de l'évolution historique de ces relations, cela implique que la conception de "monades" ou de sortes de médias "isolés" est irrecevable». Cité par A. Gaudreault, op. cit., p. 170.

3. Pour un autre regard sémiotique sur Laocoon, voir D. Wellbery, Lessing's Laocoon: Semiotics and the Age of Reason, Cambridge, Cambridge University Press, 1984.

4. G. E. Lessing, Laocoon [1766] (avant-propos de H. Damisch), Paris, Hermann, 1990.

5. C. Greenberg, "Towards a newer Laocoon" [1940], The Collected Essays of Criticism. vol. I, Perceptions and Jugdments 1939-1944 (dirigé par J. O'Brian), 1988, p. 23-39.

6. Pour une lecture attentive au XVIII e siècle et à l'avant-garde américaine, voir $O$. Asselin, «Du siège de l'âme : les déplacements du spectateur et du lecteur chez Diderot», Trois, vol. 8 n³, printemps-été 1993, p. 8-25.

7. T. de Duve, "Clement Lessing", Essais datés I - 1974-1986, Paris,

Éd. de la Différence, 1988, p. 65-117.

8. W. J. T. Mitchell, op. cit., p. 104.

9. Lessing, op. cit., p. 51.

10. Ibid., p. 58-59.

11. Ibid., p. 55-56.

12. L. Marin, Détruire la peinture, Paris, Galilée, 1977, p. 161.

13. Lessing, op. cit., p. 56.

14. Ibid., p. 49 .
15. Mitchell, op. cit., p. 112.

16. Lessing, op. cit., p. 84.

17. Mitchell, op. cit., p. 113.

18. D. Sylvester, Interwiews with Francis Bacon, Londres, Thames and Hudson, 1987, p. 34.

19. N. Dubreuil-Blondin, «Number One. Vers la construction d'un modèle ", Jackson Pollock: Questions, Montréal, Musée d'art contemporain de Montréal, 1979, p. 43-67.

20. G. Picon, «Le Cercle et le Cri», dans Francis Bacon, Paris, Centre Georges Pompidou, 1996, p. 274-275.

21. G. Deleuze, Francis Bacon. Logique de la sensation, Paris, Éd. de la Différence, 1981, p. 9 et 41-42.

22. M. Peppiatt, Anatomy of an Enigma, New York, Farrar, Straus et Giroux, 1996, p. 142.

23. Sylvester, «Un parcours», dans Francis Bacon, op. cit., 1996, p. 17.

24. Peppiatt, op. cit., p. 140.

25. Sylvester, Interviews with Francis Bacon, op.cit., p. 34-35.

26. Ibid., p. 38 .

27. S. Mariniello, «Médiation et intermédialité». Conférence prononcée à "La nouvelle sphère intermétiatique I», mars 1998, Musée d'art contemporain de Montréal. Une version de cette intervention est parue en guise de présentation du numéro "Cinéma et intermédialité ", Cinémas, vol. 10, nos 2-3, printemps 2000, p. 7-11. Mes discussions avec S. Mariniello ont été précieuses dans la révision de ce texte et je l'en remercie chaleureusement.

28. A. Ehrenberg, L'Individu incertain, Paris, Hachette, 1999, p. 25.

29. J. Russell, Francis Bacon, Paris, Thames and Hudson, 1994, p. 71. Je souligne.

30. Je remercie ici V. Rodriguez, dont la thèse de doctorat porte sur la reformulation de l'atelier, pour une intéressante discussion sur la nouvelle sociologie de la médiation.

31. "The Horrific Vision of Mr. Francis Bacon", The Times, 24 mai 1962, p. 7. Cité dans Francis Bacon, op. cit., 1996, p. 299. On trouvera dans ce catalogue un résumé de la réception critique de Bacon où se marque cet intérêt de la critique à cerner les effets de la photo et du cinéma. 